\title{
Pierre Boisard, Claude Didry et Dima Younès (dir.), Les travailleurs de l'innovation. De l'entrepreneur aux salariés
}

Rennes, Presses universitaires de Rennes, 2016, 308 p.

\section{Michel Letté}

\section{(2) OpenEdition}

\section{Journals}

\section{Édition électronique}

URL : http://journals.openedition.org/artefact/2558

DOI : 10.4000/artefact.2558

ISSN : 2606-9245

\section{Éditeur :}

Association Artefact. Techniques histoire et sciences humaines, Presses universitaires du Midi

\section{Édition imprimée}

Date de publication : 6 décembre 2018

Pagination : 390-393

ISBN : 978-2-8107-0595-5

ISSN : 2273-0753

\section{Référence électronique}

Michel Letté, «Pierre Boisard, Claude Didry et Dima Younès (dir.), Les travailleurs de l'innovation De l'entrepreneur aux salariés », Artefact [En ligne], 8 | 2018, mis en ligne le 21 juin 2019, consulté le 05 décembre 2020. URL : http://journals.openedition.org/artefact/2558; DOI : https://doi.org/10.4000/ artefact. 2558

Ce document a été généré automatiquement le 5 décembre 2020.

Artefact, Techniques, histoire et sciences humaines est mise à disposition selon les termes de la Licence Creative Commons Attribution - Pas d'Utilisation Commerciale - Pas de Modification 4.0 International. 


\title{
Pierre Boisard, Claude Didry et Dima Younès (dir.), Les travailleurs de l'innovation. De l'entrepreneur aux salariés
}

Rennes, Presses universitaires de Rennes, 2016, 308 p.

\author{
Michel Letté
}

1 Faire le lien entre différentes manières de considérer les logiques sous-jacentes aux dynamiques de production de l'innovation, telle est l'ambition d'une enquête conduite à partir de 2010 sur le travail intellectuel et les ressources matérielles mobilisées par les entreprises dites innovantes. Cet ouvrage dirigé par Pierre Boisard, Claude Didry et Dima Younès est plus précisément le bilan documenté d'un programme de recherche dont la vocation était de saisir le rôle du travail des salariés impliqués dans des trajectoires singulières d'innovation ${ }^{1}$. Le livre est la publication des actes du colloque de clôture qui s'est tenu en 2013.

2 Avant tout autre ordre de considération, les auteurs rappellent l'évidence du caractère profondément collectif de l'innovation. Celles et ceux qui y concourent sont en effet nombreux, même restreints comme ici aux salariés des entreprises concernées. Entre culte de l'entrepreneur et conviction que les ressorts de l'innovation ne peuvent être que distribués dans la masse des usagers, il y a tout un monde, celui des travailleurs. Car il y a dans le champ de la recherche et développement en entreprise des travailleurs de l'innovation comme il y a en science de la nature des travailleurs de la preuve. Tous se retrouvent néanmoins au croisement de deux certitudes. L'une est que la recherche dite fondamentale génère toujours des applications industrielles, souvent inimaginables lors de la phase d'élaboration des programmes de recherche. L'autre est que de la quête de solutions techniques imposées par les contraintes de la production peuvent bien tout autant émerger des connaissances théoriques tout aussi fondamentales. 
3 De ces oppositions savamment cultivées au XIX siècle entre "fondamentalistes » et «utilitaristes » est apparue l'innovation au siècle suivant comme la solution à tous les problèmes, jusqu'à devenir le mantra de toute entreprise et de toute politique publique de développement économique. L'intérêt porté à toutes celles et ceux qui par-delà l'entrepreneur contribuent aux processus de production de l'innovation s'est accru d'autant. Le présent ouvrage est une de ces contributions parmi les plus récentes visant à éclairer les zones d'ombre produites par la lumière parfois aveuglante du génie individuel.

4 Au travers de dix-sept études de cas de trajectoires singulières d'une ou plusieurs innovations, chacune des contributions tente d'accéder à la texture fine des identités, des rôles, des fonctions et des liens entre lesdits travailleurs de l'innovation. Elles rendent ainsi en partie justice aux agissants dans l'ombre de l'entrepreneur emblématique, sans pour autant les dissocier du réseau des contraintes dans lequel tout ce monde du travail est pris constamment.

5 Quel que soit l'angle d'attention qu'on lui porte, les auteurs rappellent ainsi que l'innovation en entreprise est toujours structurée par des tensions fortes entre les activités d'exploration et d'exploitation, entre logiques d'internalisation et d'externalisation de la recherche en lien avec la production. Aucune entreprise n'échappe aux dilemmes que ces tensions recouvrent. De même, les processus d'innovation sont toujours pris dans des agencements complexes d'entreprises et d'entrepreneurs, de stratégies d'investissements et de politiques publiques.

6 Aucune trajectoire d'innovation ne ressemble tout à fait à une autre. L'un des résultats suggéré dans cet ouvrage est que ces difficultés sont en partie surmontées au travers des liens durables établis entre les salariés des différentes entités constituant des collectifs de travail, des communautés de pratiques et de savoirs. Les logiques sousjacentes aux dynamiques d'innovation s'appuient ainsi toujours sur les travailleurs qui les incarnent, sur leurs actions, que ces dernières soient d'ailleurs de résistance ou de coopération.

7 Une fois dit tout cela, il restait à identifier qui sont ces travailleurs de l'innovation tels qu'annoncés dans le titre. La façon de les désigner est importante. Elle considère ici les travailleurs que la quête de solutions nouvelles engage. Recherche de solution nouvelle, l'innovation mobilise en conséquence des collectifs de travail dédiés. Sans exclure totalement les pratiques d'adaptation technique et d'ajustement du comportement des salariés dans toute entreprise de production, l'innovation dite ordinaire n'est pas cependant celle que privilégient ici les auteurs. Les travailleurs de l'innovation sont dans cet ouvrage plutôt ceux explicitement et directement impliqués par le projet institué, quels que soient son cadre de réalisation et l'espace délimité de production de l'innovation.

8 On aurait pu dès lors s'attendre à trouver dans ce livre quelques parcours individuels de salariés parmi ces travailleurs tels que désignés précédemment. En fait il n'en est rien. Les travailleurs de l'innovation renvoient pour l'essentiel à des catégories d'acteurs plutôt qu'à des individus singuliers: des chercheurs, des ingénieurs, des techniciens, des ouvriers, des salariés des services juridiques et des managers, considérés comme collectifs plus ou moins anonymes. Les quelques individus interviewés restent surtout proches de l'entrepreneur individuel ou du haut cadre responsable de la direction de l'innovation (tel Jean-Luc Beylat pour les Bell Labs en 
France) que la multitude des travailleurs, certes plus discrète, mais au rôle non moins déterminant dans la conduite du processus d'innovation sur le terrain.

La remarquable contribution de Tommaso Pardi sur la passion de l'innovation portée par les ingénieurs du constructeur Renault est particulièrement éclairante sur ce point. L'histoire du travail d'innovation engagé autour du moteur à deux temps est passionnante mais d'abord restituée sur le mode du réseau des contraintes économiques et des impératifs organisationnels au sein du groupe et de la concurrence internationale. Les structures et les dispositifs institutionnels comptent d'abord, déterminés par des questions de stratégies portées par les décideurs et donneurs d'ordres. Presque en filigrane, des collectifs de personnes, notamment des équipes d'ingénieurs, font advenir des promesses de résultats, des prototypes, mais surtout assurent un rôle décisif dans la pérennisation des potentiels d'innovation que les entrepreneurs $\mathrm{du}$ groupe industriel activent, déplacent ou effacent au fil des opportunités.

L'ensemble des contributions renvoie ainsi à des espaces de travail plutôt qu'aux travailleurs eux-mêmes. Il est au demeurant bien difficile de définir a priori une catégorie spécifique de travailleurs de l'innovation. Peu importe. La lecture de cet ouvrage clair offre un paysage de l'innovation d'une grande richesse au travers de l'épaisseur des trajectoires d'innovation explorées, des intrications complexes et modèles de coordination entre institutions, collectifs et individus. Toujours différentes, toujours singulières, elles sont toutes portées par des collectifs de travail, des communautés de pratiques, de partenariats, de systèmes locaux de compétences, de dispositifs de gouvernance, de réseaux de personnes et de connaissances, bref par des collectifs parmi de nombreux autres et façon de désigner ces différents espaces d'échanges entre travailleurs de l'innovation.

11 On pourrait vite se perdre dans le méandre des parcours et modèles de production de l'innovation. Ce livre permet de s'y retrouver un peu. Utile à la réflexion, il invite à bien des réflexions, contribue à faire se poser d'autres questions, suggère des grilles de lecture et d'analyse des fonctions que les travailleurs de l'innovation assurent par-delà le rôle de l'entrepreneur individuel. L'innovation n'a jamais seulement été une affaire de génie individuel. Une belle démonstration.

\section{NOTES}

1. « Portrait du chercheur en travailleur salarié », projet réalisé dans le cadre du programme AnR «Sciences, technologies et savoirs en société. Enjeux actuels, questions historiques» (AnR Travcher). 


\section{AUTEUR}

\section{MICHEL LETTÉ}

Conservatoire national des arts et métiers 